\title{
An improved DAC driving scheme for OFDM applications
}

Benoit Catteau, Pieter Rombouts and Ludo Weyten

This document is an author's draft version submitted for publication in Electronics Letters

The actual version was published as:

B. Catteau, P. Rombouts, and L. Weyten, "Improved DAC driving scheme for OFDM applications," Electronics Letters, vol. 46, no. 16, pp. 11031104, Aug 5, 2010. 


\title{
An improved DAC driving scheme for OFDM applications
}

\author{
B. Catteau, P. Rombouts and L. Weyten
}

\begin{abstract}
We present a "Redundant Signed Digit" (RSD) driving scheme for binary weighted $D / A$ conversion. This scheme retains the simplicity of conventional binary weighted D/A conversion, but has greatly improved robustness with regard to parasitic effects, especially when processing signals with a high crest factor. Such signals occur in communication systems that use e.g. orthogonal frequency-division multiplexing (OFDM). In this case we measured an improvement of $9 \mathrm{~dB}$ in Missing Tone Power Ratio performance on a prototype $250 \mathrm{MSample} / \mathrm{s}$ circuit implemented in $0.18 \mu \mathrm{m}$ CMOS.
\end{abstract}

Introduction Orthogonal frequency-division multiplexing (OFDM) is frequently used in contemporary communication systems such as XDSL and next generation systems such as LTE [1]. The associated signals have a large crest factor and thus, small signal values occur much more often than large signal values. This way, the D/A converter (DAC) must maintain an acceptable signal integrity for both small and large input signals. In many cases this leads to a requirement of 12 effective bits for the DAC, which translates to sophisticated and areaconsuming circuits [1]. A widely adopted approach to obtain this performance is the use of thermometer encoding for the most significant bits in the DAC [1]. However, for design simplicity (which reduces design time and silicon area) a more preferable structure would only use binary weighted elements. Unfortunately, in such a binary weighted structure the largest (i.e. MSB) element toggles quite often, also for small amplitude signals. Each time this MSB element is toggled, its associated dynamic and static error is injected into the output signal, hereby severely affecting the signal integrity. As a result a very poor signal integrity is obtained for small signals. In this work, we use the Redundant Signed Digit (RSD) coding scheme for binary weighted D/A conversion. This technique originates from the Analog to 
Digital domain [2] and is applied here to D/A conversion. It will be demonstrated that this approach greatly improves the DAC performance for OFDM-like signals.

RSD Coded DAC The architecture of an RSD-DAC will be explained here starting from a classic binary weighted DAC. A digital input word $D_{i n}$, which consists of $N$ bits, needs to be converted into an analog signal. Without loss of generality we normalize $D_{i n}$ such that all valid values of $D_{i n}$ are within the interval $(-1,+1)$. Now, a straightforward way to perform this operation is to use a binary weighted current-steering DAC. For this conversion $N$ binary weighted current sources are used. It follows that the selection signals, $S_{j}$, needed to control the switches in the DAC, can directly be derived from the bits of the input word $D_{i n}$, where $-1<D_{\text {in }}<1$ and $S_{j}$ can be 1 or -1 . Depending on the value of $S_{j}$, the current $I_{j}$ of source $j$ will flow into one of the matched output resistors $R_{L}$ or $R_{L}^{\prime}$ (Fig. 1(a)). This way, the differential voltage signal, $V_{\text {out }}$ produced at the output of the DAC will be proportional to $D_{\text {in }}$ and equal to

$$
V_{\text {out }}=R_{L} \sum_{j=1}^{N} S_{j} I_{j}
$$

For a binary weighted DAC this can be simplified to

$$
V_{\text {out }}=R_{L} I_{1} \sum_{j=1}^{N} S_{j} 2^{j-1}
$$

where $I_{1}$ is the current of the smallest current cell.

[Figure 1 about here.]

Instead of using the bits of the digital word directly, the $S_{j}$ can also be derived from a cascade of selection logic stages (Fig. 1(b)). While this approach seems an unnecessary complication for binary weighted DAC's, its generalization for RSD-DACs will prove useful in this discussion. This way, every selection signal $S_{j}$ is made in a separate stage $j$ of the pipeline, based on the 
input $D_{j}$,

$$
S_{j}=\left\{\begin{array}{c}
1 \quad \text { when } 0 \leq D_{j} \leq 1 \\
-1 \quad \text { when }-1 \leq D_{j} \leq 0,
\end{array}\right.
$$

Here $D_{j}$ denotes the input signal of stage $j$. The output (or input of the next stage) $D_{j+1}$ becomes

$$
D_{j+1}=2 D_{j}-S_{j}
$$

The input-output characteristic of one stage of this binary selection logic is shown in Fig. 2(a). In order to implement an RSD-DAC now the selection signal is also allowed to be equal to 0 , and the decision rule changes into

$$
S_{j}=\left\{\begin{array}{cl}
1 & \text { when } \alpha \leq D_{j} \leq 1 \\
0 & \text { when }-\alpha<D_{j}<\alpha \\
-1 & \text { when }-1 \leq D_{j} \leq-\alpha,
\end{array}\right.
$$

where $\alpha$ denotes the decision level. While in most situations where RSD coding is used $\alpha$ is equal to 0.25 [2], in reality the coding works for any value $0 \leq \alpha \leq 0.5$. This way, three states are possible for every stage, while the output of one stage is still defined by (1). The input-output characteristic is shown in Fig. 2(b).

\section{[Figure 2 about here.]}

The use of RSD coding in the selection logic implies a slight modification of the normal current-steering DAC, to handle the three-state selection signal $S_{j}$. A simple solution for this is to implement two half-weight current sources for each RSD coded bit. The area for the current sources is not increased by this rearrangement and it is clear that such a circuit can deliver a differential current equal to $0,+I_{j}$ or $-I_{j}$ to the load and thus the three RSD-states can be created. Let us now illustrate how this coding scheme improves the performance for signals with a high crest factor. This means that those signals contain a lot of small values 
and very few large values. By introducing a state $S_{j}=0$ for every stage of the RSD-DAC and knowing that inputs generally have a mean value equal to zero, the number of switching current sources is adjusted to the input signal level. Indeed, when an input with a small value, e.g. $\alpha / 3$, is applied to the RSD-DAC, the first stage will output a selection signal $S_{1}$ equal to 0 and a residue signal equal to $2 D_{1}$. The second stage will show a selection signal $S_{2}$ which equals to 0 and an output signal equal to $4 D_{1}$, see Fig. 3 . This means that the first two stages that contain the largest current sources, will not be used when this signal is converted into an analog current. This way their associated static and dynamic errors will not be injected in the signal, only the errors of the remaining stages will be injected, but these are a lot smaller than those of the first stages.

[Figure 3 about here.]

Experimental Results The concept was verified through various computer simulations on OFDM signals. In this application the performance is quantified by the Missing Tone Power Ratio (MTPR). In the simulation an improvement in MTPR of about $12 \mathrm{~dB}$ was obtained by using RSD coding. Also actual experimental measurements were performed. For this purpose the analog portion of a current steering DAC was integrated in a $0.18 \mu \mathrm{m}$ CMOS chip with an active area of $0.1 \mathrm{~mm}^{2}$. The intrinsic matching of the current sources was as low as 8-bit. For ease of experimentation, the digital RSD-coder was not co-integrated in the ASIC, but instead implemented in an external FPGA. Also a digital OFDM-generator with 12-bit resolution was implemented in the FPGA. Due to this set-up, the maximum clock frequency was limited to $250 \mathrm{MHz}$, which is used in these measurements. The upstream band goes from $5 \mathrm{MHz}$ to $15 \mathrm{MHz}$, and the downstream band from $20 \mathrm{MHz}$ to $50 \mathrm{MHz}$. In the test signal, 96 carriers are used and the crest factor was $1 / 6$. The full scale output current was set to $20 \mathrm{~mA}$. The resulting output signal was connected to a spectrum analyzer for evaluation. 
[Figure 4 about here.]

In order to evaluate the effectiveness of the RSD scheme the converter was tested for both the case of RSD coding and conventional binary weighted coding, which was implemented as well. For the RSD coding the transition levels $\pm \alpha$ where set at -0.5 and 0.5 . Fig. 4 shows the corresponding output spectra. In the case of binary weighted coding, the MTPR equals $41 \mathrm{~dB}$ while the MTPR for RSD coding is $50 \mathrm{~dB}$, which is an improvement of $9 \mathrm{~dB}$.

Conclusions In this paper, we have presented a DAC circuit which employs a "Redundant Signed Digit" (RSD) coding scheme to drive an array of binary weighted sources. This scheme greatly improves the performance for small signals. This way this structure is especially useful for signals with a large crest factor such as OFDM signals. Here we measured an improvement in MTPR of $9 \mathrm{~dB}$ on an experimental $250 \mathrm{MS} / \mathrm{s}$ prototype which was integrated on a very small silicon area of only $0.1 \mathrm{~mm}^{2}$ of silicon area in $0.18 \mu \mathrm{m}$ CMOS.

Authors' affiliations: B. Catteau, P. Rombouts and L. Weyten, Electronics and Information Systems Department, Ghent University, St. Pietersnieuwstraat 41, B-9000 Gent, Belgium

\section{References}

[1] W. De Wilde, N. Scantamburlo, M. Combe, J. Van Leeuwe, K. Doorakkers, Y. Mazoyer, C. Renous, R. Petigny, A. Bonin, B. Bayracki, B. Belhi, E. Moons, and J. Sevenhans, "Analog front end for dmt-based vdsl," in Digest of Tech. Papers of the IEEE Int. SolidState Circuits Conf. 2002, vol. 1, pp. $328-471$.

[2] B. Ginetti, P. Jespers, and A. Vandemeulebroecke, "A CMOS 13-b Cyclic RSD A/D Converter," IEEE J. Solid-State Circuits, vol. 27, no. 7, pp. 957-965, 1992. 


\section{List of Figures}

1 DAC-structure: (a) analog part and (b) digital part. . . . . . . . . . . 7

2 The input-output characteristics of a binary stage (a) and an RSD-stage (b). . 8

3 The RSD stages for an input with amplitude equal to $\alpha / 3 \ldots \ldots . . . . .9$

4 Output spectrum for an OFDM input signal with (a) conventional binary weighted coding and (b) RSD coding $(\alpha=0.5) \ldots \ldots \ldots 10$ 


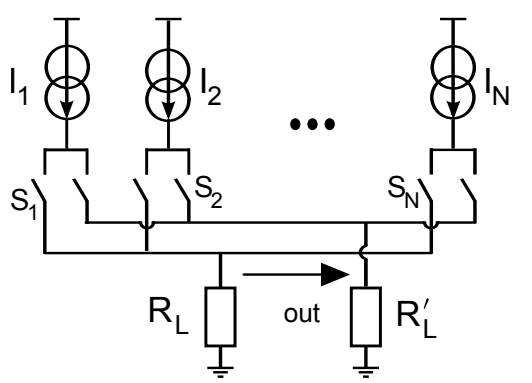

(a)

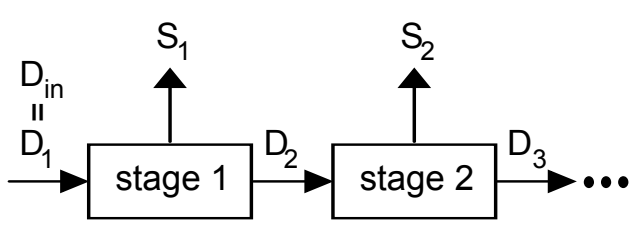

(b)

Figure 1: DAC-structure: (a) analog part and (b) digital part. 


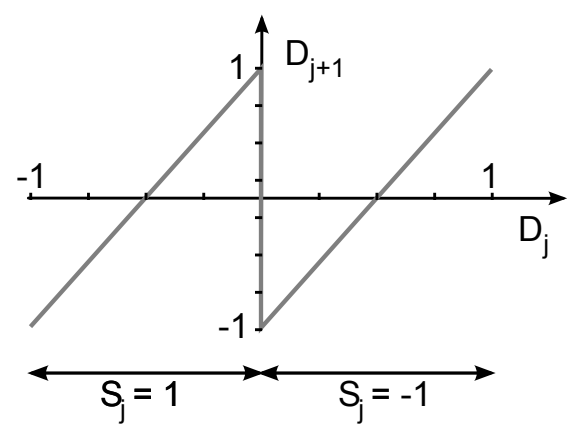

(a)

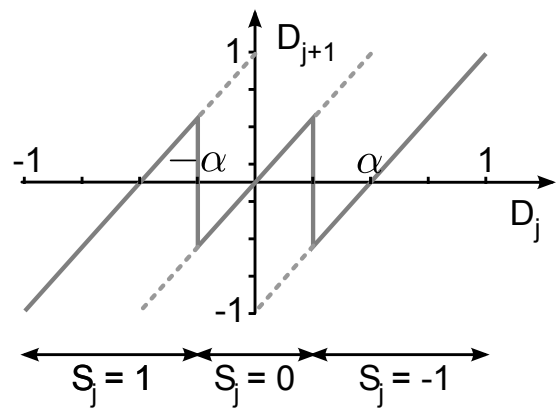

(b)

Figure 2: The input-output characteristics of a binary stage (a) and an RSD-stage (b). 

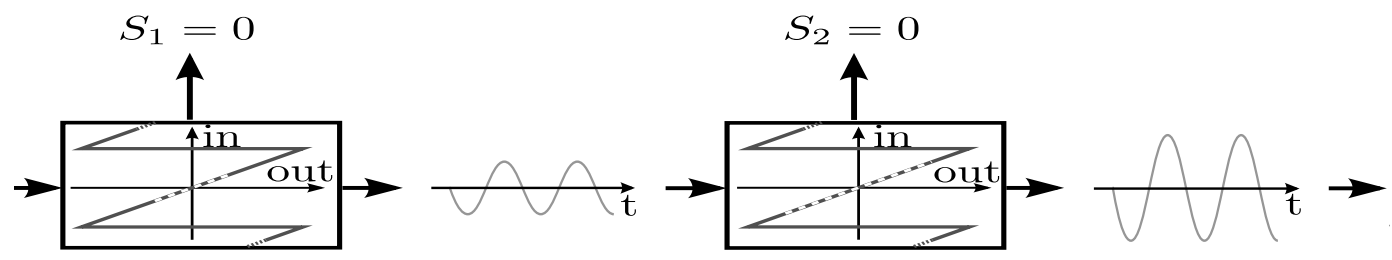

Figure 3: The RSD stages for an input with amplitude equal to $\alpha / 3$ 


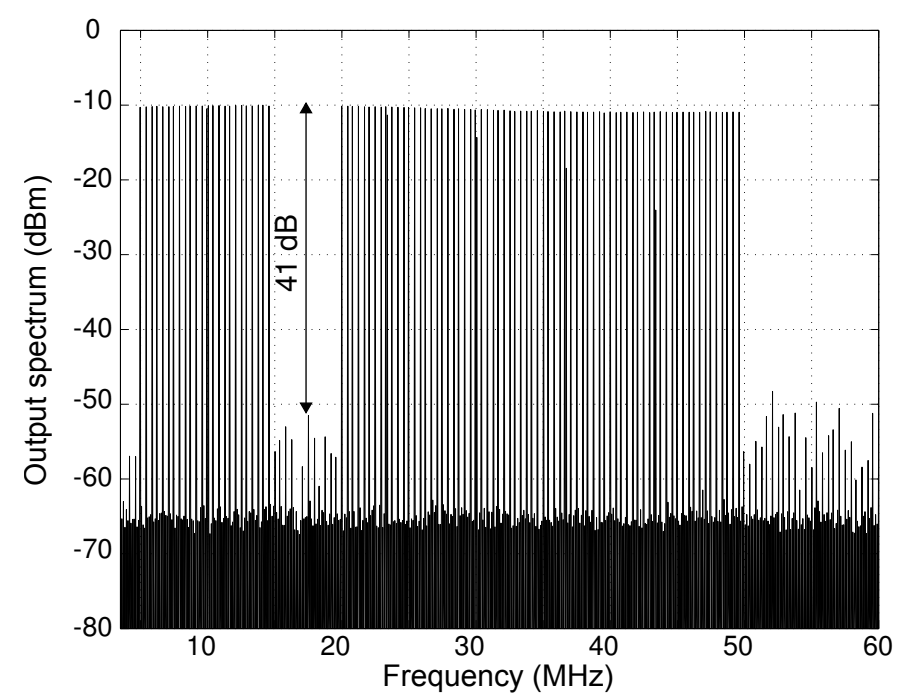

(a)

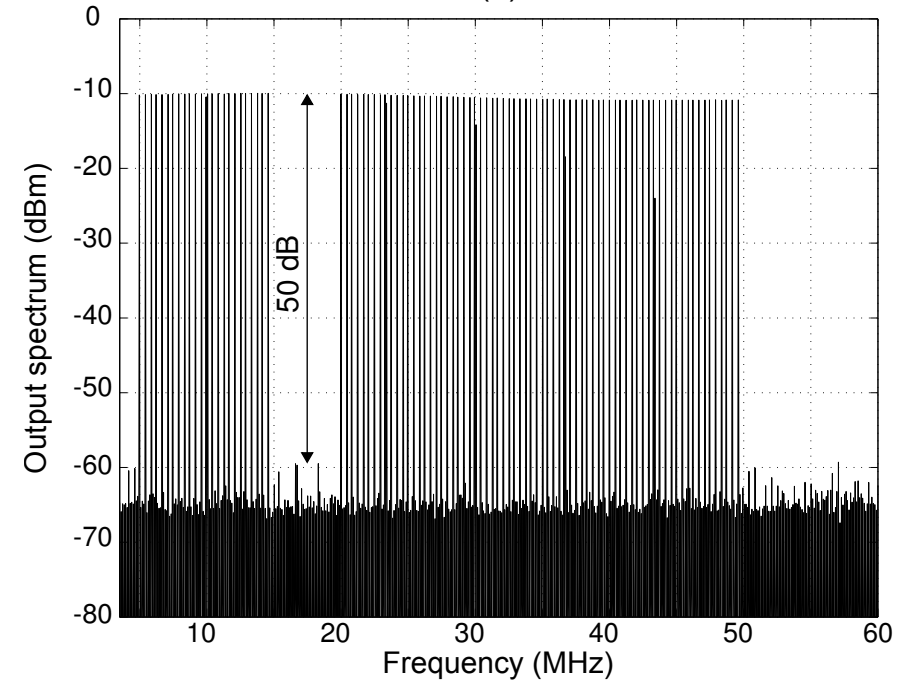

(b)

Figure 4: Output spectrum for an OFDM input signal with (a) conventional binary weighted coding and (b) RSD coding ( $\alpha=0.5)$. 